\title{
$\mathrm{M}|\mathrm{R}| \mathrm{S}$ Internet Journal Nitride Semiconductor Research
}

\section{Visible-Blind UV Digital Camera Based On a 32 x 32 Array of GaN/AIGaN p-i-n Photodiodes}

\author{
J.D. Brown ${ }^{1}$, Zhonghai Yu ${ }^{1}$, J. Matthews ${ }^{1}$, S. Harney ${ }^{1}$, J. Boney ${ }^{1}$, J.F. Schetzina ${ }^{1}$, J.D. Benson ${ }^{3}$, \\ K.W. Dang ${ }^{3}$, C. Terrill ${ }^{3}$, Thomas Nohava ${ }^{2}$, Wei Yang ${ }^{2}$ and Subash Krishnankutty ${ }^{2}$ \\ ${ }^{1}$ Department of Physics, North Carolina State University, \\ ${ }^{2}$ Honeywell Technology Center, \\ ${ }^{3}$ Night Vision Laboratory, Ft. Belvoir, VA,
}

(Received Wednesday, August 25, 1999; accepted Friday, September 3, 1999)

A visible-blind UV camera based on a 32 x 32 array of backside-illuminated GaN/AlGaN p-i-n photodiodes has been successfully demonstrated. Each of the 1024 photodiodes in the array consists of a base n-type layer of $\mathrm{AlGaN}(\sim 20 \%)$ onto which an undoped $\mathrm{GaN}$ layer followed by a p-type $\mathrm{GaN}$ layer is deposited by metallorganic vapor phase epitaxy. Double-side polished sapphire wafers are used as transparent substrates. Standard photolithographic, etching, and metallization procedures were employed to obtain fully-processed devices. The photodiode array was hybridized to a silicon readout integrated circuit using In bump bonds. Output from the UV camera was recorded at room temperature at a frame rate of $30 \mathrm{~Hz}$. This new type of visible-blind digital camera is sensitive to radiation from $320 \mathrm{~nm}$ to $365 \mathrm{~nm}$ in the UV spectral region.

\section{Introduction}

The rapid development of device quality $\mathrm{GaN}$, InGaN and AlGaN over the past five years has lead to the commercialization of blue and green light emitting diodes, along with demonstrations of violet laser diodes and a variety of electronic devices [1] [2] [3] [4] [5] [6] [7] [8] [9] [10]. In addition, a number of photodetectors based on photoconductive and junction devices have also recently been reported [11] [12] [13].

This paper reports the first successful demonstration of an ultraviolet (UV) digital camera based on an array of GaN/AlGaN heterostructure $\mathrm{p}-\mathrm{i}-\mathrm{n}$ photodiodes. This visible-blind ( $32 \times 32$ pixel) digital imager is designed to sense radiation only in the $320-365 \mathrm{~nm}$ wavelength band in the UV spectral region. In this paper, a general discussion of photodiode properties is followed by a description of the experimental procedures employed to synthesize, process and study discrete photodiodes and photodiode arrays. This is followed by a discussion of the experimental results obtained.

When fully developed, this new type of UV digital camera may be expected to be used in many wide-ranging applications including welding imagery, flame sensing, biological agent detection, missile and shellfire detection, and in studies of astronomical objects.

\section{Properties of Semiconductor Photodiodes}

Let us consider a $\mathrm{p}-\mathrm{n}$ junction or $\mathrm{p}-\mathrm{i}-\mathrm{n}$ junction photodiode operating in the photovoltaic mode at zero bias [14]. The detector current responsivity $R_{\lambda}$ is defined as the output current $i_{p}$ produced by the detector divided by the diode illumination power $\mathrm{P}_{\lambda}$ at a given wavelength $\lambda$. That is,

$$
\mathrm{R}_{\lambda}=\mathrm{i}_{\mathrm{p}} / \mathrm{P}_{\lambda}=\mathrm{q \eta} / \mathrm{hv} \quad(\mathrm{A} / \mathrm{W})
$$

where $\mathrm{q}$ is the electronic charge, $\eta$ is the quantum efficiency, $h$ is Planck's constant and $v$ is the radiation frequency. Note that the responsivity $R_{\lambda}$ varies inversely with the optical frequency $v$. As a consequence, maximum responsivities $(\eta=1)$ in the UV are small — ranging from $R_{\lambda}=0.294 \mathrm{~A} / \mathrm{W}$ at $365 \mathrm{~nm}$ to $R_{\lambda}$ $=0.161 \mathrm{~A} / \mathrm{W}$ at $200 \mathrm{~nm}$.

An important figure of merit for a diode detector is the $\mathrm{R}_{0} \mathrm{~A}$ product, where $\mathrm{R}_{0}$ is the dynamic resistance of the diode at zero bias and $\mathrm{A}$ is the detector area. A large $\mathrm{R}_{0} \mathrm{~A}$ product is a necessary requirement for the detector to have a large detectivity $\mathrm{D}^{*}$, that is, to be able to detect very low light levels. Figure 1 shows the spectral detectivity $\mathrm{D}^{*}$ for a number of different commercially-available detectors [15] that operate at wavelengths ranging 
from $0.2 \mu \mathrm{m}$ (UV) to $12 \mu \mathrm{m}$ (IR). It is seen from the figure that IR detectors have $D^{*}$ values of $10^{7}-10^{10}(\mathrm{~cm}$ $\mathrm{Hz}^{1 / 2} \mathrm{~W}^{-1}$ ) depending on the type of detector, its operating temperature, and the wavelength region for which it is designed. Detectors operating at shorter wavelengths generally have larger $D^{*}$ values ranging up to $D^{*}=4 x$ $10^{14}\left(\mathrm{~cm} \mathrm{~Hz}^{1 / 2} \mathrm{~W}^{-1}\right)$ for a photomultiplier tube (PMT) operating at about $300 \mathrm{~nm}$.

Figure 1 also shows the curve for an ideal photovoltaic detector limited by thermal background radiation from a 293K radiation source (the earth!!). A detector displaying $\mathrm{D}^{*}$ values that lie along this line is said to be background limited. A Background LImited Photodetector is often referred to as being a BLIP-limited detector, or as being BLIP-limited [14]. These detectors have the highest possible $\mathrm{D}^{*}$ values for a given wavelength when exposed to the earth's thermal radiation field. Note from the figure, that $\mathrm{D}^{*}$ values for a BLIPlimited detector become extremely large as the wavelength decreases into the UV. This is because a $293 \mathrm{~K}$ black body radiation background contains virtually no UV radiation, so an extremely sensitive detector is required to be able to be detect this near-zero thermal background from the earth.

The spectral detectivity $\mathrm{D} *$ of a photodiode detector may be expressed as [14]

$$
D^{*}=(q \eta / h v)\left[\left(4 k T / R_{0} A\right)+2 q^{2} \eta \Phi_{b}\right]^{-1 / 2} \quad\left(c m ~ H z z^{1 / 2} W^{-1}\right)
$$

The first term in the bracketed quantity above (4kT/ $\mathrm{R}_{0} \mathrm{~A}$ ) arises from thermal noise sources within the detector. The second term $\left(2 q^{2} \eta \Phi_{b}\right)$ is due to the background radiation $\Phi_{\mathrm{b}}$ to which the detector is exposed. If the detector is limited by thermal noise, that is, if the thermal noise far exceeds the background radiation induced signal, then the detectivity becomes

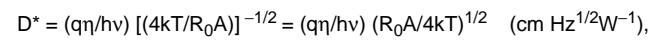

where $\mathrm{k}$ is Boltzmann's constant and $\mathrm{T}$ is the absolute temperature. Note that the detectivity $\mathrm{D}^{*}$ is directly proportional to $\left(\mathrm{R}_{0} \mathrm{~A}\right)^{1 / 2}$ when the detector is noise-limited. Thus, to develop very sensitive detectors it is essential that the detector noise sources be minimized so that $\mathrm{R}_{0} \mathrm{~A}$ is large. In the case of III-V nitrides, the extremely large density of dislocations represents a significant noise source. Therefore, dislocation reduction should have a very positive effect on the detectivity of nitride photodiodes.

If the second term in brackets of equation (2) above dominates, then the background signal determines $\mathrm{D}^{*}$.
In this case, the detectivity $\mathrm{D}^{*}$ is BLIP-limited and may be expressed as

$$
D^{*}{ }_{\text {BLIP }}=(q \eta / h v)\left[2 q^{2} \eta \Phi_{b}\right]^{-1 / 2}=(1 / h v)\left(\eta / 2 \Phi_{b}\right)^{1 / 2}\left(\mathrm{~cm} \mathrm{~Hz}^{1 / 2} W^{-1}\right) .
$$

The detectivity D* may also be expressed in terms of the detector noise equivalent power NEP such that [14]

$$
D^{*}=(A \Delta f)^{1 / 2} / N E P \quad\left(\mathrm{~cm} \mathrm{~Hz}^{1 / 2} \mathrm{~W}^{-1}\right)
$$

where $\mathrm{A}$ is the detector area and $\Delta \mathrm{f}$ is the detector bandwidth. The noise equivalent power NEP is the power that corresponds to the incident rms optical power required such that the detector signal-to-noise ratio is one in a bandwidth of $1 \mathrm{~Hz}$.

\section{Experimental Details}

The photodiode structure employed in the present work is shown schematically in Figure 2. It consists of a base layer of $\mathrm{n}-\mathrm{AlGaN}(\sim 20 \% \mathrm{Al})$ followed by an undoped $\mathrm{GaN}$ layer and a $\mathrm{p}-\mathrm{GaN}$ layer. The photodiode structure is deposited by MOVPE onto a polished sapphire wafer to permit illumination of the device through the substrate. As shown in the figure, the photodiode responds to UV light in the wavelength band from about $320 \mathrm{~nm}$ to $365 \mathrm{~nm}$. At wavelengths shorter than $320 \mathrm{~nm}$, the incoming light is absorbed in the thick AlGaN base layer and the junction is not illuminated. Likewise, the diode does not respond to wavelengths greater than 365 $\mathrm{nm}$, since this corresponds to the optical absorption edge of $\mathrm{GaN}$ at $300 \mathrm{~K}$. By increasing the $\mathrm{Al}$ content of the base layer it is possible to increase the optical bandwidth of the diode's UV responsivity. Likewise, by adding $\mathrm{Al}$ to the top layers, it is possible to change the diode UV responsivity band to other wavelength regions in the UV. Thus, UV detectors that sense different UV "colors" are possible.

Diode structures of the type shown were prepared by MOVPE both at North Carolina State University (NCSU) and at the Honeywell Technology Center using low-pressure, vertical-flow MOVPE reactors that employ high speed substrate rotation during film growth. The photodiode structures were deposited onto 2 in diameter c-plane sapphire substrates. The growth was initiated by depositing a thin AIN buffer layer at 500-650 ${ }^{\circ} \mathrm{C}$; all subsequent layers were grown at 1050$1080^{\circ} \mathrm{C}$.

All device processing was completed at NCSU using standard semiconductor processing techniques which included photolithography using appropriately-designed masks, reactive ion etching to define mesa structures, and metallizations to provide ohmic contacts to the ntype and p-type layers of the device. 
Spectral responsivity measurements were completed at NCSU on selected discrete photodiodes. In these experiments a xenon lamp was employed as a UV source and the wavelengths were selected by a monochromator. The monocromator output was calibrated using a calibrated UV-enhanced Si photodiode. The $300 \mathrm{~K}$ dynamic resistance of the photodiode at zero-bias $\mathrm{R}_{0}$ was measured for selected devices using a shielded low-noise enclosure and shielded probe tips. These measurements were combined with the device area A to obtain the $\mathrm{R}_{0} \mathrm{~A}$ product. Equation (3) was then used to obtain an estimate of the detector detectivity, $\mathrm{D}^{*}$.

In order to use the GaN/AlGaN photodiodes as the basis for a new visible-blind UV digital camera it is necessary to employ flip-chip bonding techniques to hybridize an appropriately sized photodiode array to a silicon readout integrated circuit (ROIC) as shown in Figure 3. In the present case, NCSU designed and purchased a mask set for a photodiode array that matched a $32 \times 32$ ROIC chip provided by the Night Vision Laboratory (NVL) at Ft. Belvoir. A fully processed 32 x 32 photodiode array is shown in Figure 4. The array consists of 1024 mesa diodes of area $44 \mu \mathrm{m}$ x $44 \mu \mathrm{m}$ on a $53.4 \mu \mathrm{m}$ pitch. Note from the figure that n-type metal contacts extend between all 32 rows of the array to insure a short current path for each pixel when the photodiode array is illuminated.

The Si ROIC device employed in the hybridization process is shown in Figure 5. Prior to hybridization at NVL, In bumps were deposited onto each of the mesas and $\mathrm{n}$-contact layers of the photodiode array and onto the corresponding areas of the ROIC using NCSU facilities. Each $32 \times 32 \mathrm{GaN} / \mathrm{AlGaN}$ photodiode array was hybridized to the silicon ROIC using facilities at NVL and then cemented onto a leadless chip carrier. Gold wire bonds were then used to link the various input/output channels from the ROIC to the leadless chip carrier. A closeup of a hybridized UV photodiode array is shown in Figure 6 mounted onto a printed circuit board in preparation for testing.

The experimental setup at NVL that was used for testing the GaN/AlGaN UV FPA is shown in Figure 7. It consists of an alpha-numeric UV source, a quartz focusing lens, the UV digital camera, and a digital camera computerized imaging system. An Oriel model 6035 $\mathrm{Hg}(\mathrm{Ar})$ pencil lamp fitted with a 6042 flourescent filter was used as a UV light source. This produced a rectangular $(0.5 \mathrm{~cm} \times 3 \mathrm{~cm}) \mathrm{UV}$ output centered at about 350 $\mathrm{nm}$ with a FWHM of $\sim 40 \mathrm{~nm}$. In order to provide an alpha-numeric UV scene, a thin sheet of brass was used to mill out the letters "NCSU". This brass aperture was then attached to the front of the UV source as shown in Figure 8 to provide a UV back-lighted scene. A fused quartz lens of focal length $25 \mathrm{~mm}$ was used to focus the UV scene onto the GaN/AlGaN FPA as shown in Figure 7.

The output from the visible-blind UV camera was connected to an Amber Proview imaging system. The Proview system contains electronics to support the imaging of FPA devices and to interface FPA devices to digital recording and processing equipment. In addition, it can convert the digital data into an RS-170 NTSC analog video format that can be displayed on any RS170 compatible monitor.

\section{Results and Discussion}

\subsection{Discrete GaN/AIGaN p-i-n Heterostructure Pho- todiode}

The room temperature spectral responsivity of a discrete GaN/AlGaN p-i-n heterostructure photodiode is shown in Figure 9. This particular device, which was obtained from the same wafer as the FPA, consists of a base ntype layer of $\mathrm{Al}_{0.2} \mathrm{Ga}_{0.8} \mathrm{~N}$, an undoped $\mathrm{GaN}$ layer, and a p-type GaN:Mg layer deposited by MOVPE. It is seen from the figure that the device has a sharp cut-on beginning at about $365 \mathrm{~nm}$, which corresponds to the optical absorption edge of $\mathrm{GaN}$ at room temperature. The responsivity reaches its maximum value of $0.2 \mathrm{~A} / \mathrm{W}$ at a wavelength of $358 \mathrm{~nm}$. According to equation (1), this corresponds to an external quantum efficiency of $70 \%$. Assuming the reflection losses at the air-sapphire, sapphire-AlGaN, and AlGaN-GaN interfaces are about 12 $\%$ [12], one obtains an internal quantum efficiency of about $82 \%$ for this device. The responsivity remains fairly constant until about $320 \mathrm{~nm}$ and then begins to decrease rapidly as optical absorption occurs in the $\mathrm{Al}_{0.2} \mathrm{Ga}_{0.8} \mathrm{~N}$ base layer of the device.

Figure 10 shows plots of the current-voltage (I-V) characteristics and the dynamic resistance $\mathrm{dV} / \mathrm{dI}$ versus voltage for the discrete device. It is seen from the figure that the dynamic resistance peaks close to zero-bias, indicating that the device should be most sensitive to UV radiation when operated in the photovoltaic mode at or near zero-bias. Using the device area $\mathrm{A}$ along with Equation (3) one obtains $\mathrm{R}_{0} \mathrm{~A}=1.5 \times 10^{9} \Omega-\mathrm{cm}^{2}$ and $\mathrm{D}^{*}=6.1 \times 10^{13} \mathrm{~cm} \mathrm{~Hz}^{1 / 2} \mathrm{~W}^{-1}$ for this discrete photodiode. This $\mathrm{D}^{*}$ value, obtained at room temperature, is one of the largest $\mathrm{D}^{*}$ values ever obtained for any semiconductor photodetector operating at any temperature, as is illustrated by the $\mathrm{D}^{*}$ values for various devices shown in Figure 1. Note from the figure that the GaN/ AlGaN p-i-n photodiode $D^{*}$ value of $6.1 \times 10^{13} \mathrm{~cm}$ $\mathrm{Hz}^{1 / 2} \mathrm{~W}^{-1}$ is more than an order of magnitude higher that D* values for UV-enhanced Si photodiodes at $358 \mathrm{~nm}$, and is within a factor of five of $\mathrm{D}^{*}$ values associated 
with commercial UV-enhanced photomultiplier tubes at this wavelength.

\subsection{GaN/AIGaN 32 x 32 Photodiode Array}

Figure 11 shows the output seen on the Proview system monitor on July 28, 1999 when the GaN/AlGaN photodiode array was initially illuminated with UV radiation from the "NCSU" UV source. This represents the first demonstration of a visible-blind UV camera based on an array of III-V nitride p-i-n photodiodes. Figure 12 shows a direct comparison of the UV source and the UV digital image of the source obtained via the GaN/AlGaN FPA. It should be pointed out that the digital images shown represent raw data from the nitride FPA. No attempt was made to enhance the image, although the Proview imaging system has provision for several image processing functions such as non-uniformity correction, global gain and offset, pixel de-select, and pixel replacement. Applying these procedures would produce a cleaner, more uniform digital image. The images shown were obtained with the photodiode biases set at $-0.1 \mathrm{~V}$, the pixel integration time set at 32 milliseconds, and the camera frame rate set at $30 \mathrm{~Hz}$. It should also be noted that to prevent pixel saturation under these conditions, with the UV source positioned to produce the UV images shown, it was necessary to employ neutral density filters (ND 1) to decrease the UV flux at the FPA. This clearly demonstrates that the FPA responsivity is more than adequate to obtain high quality UV images -even under low light conditions.

By moving the UV source up and down and to the right and left relative to the stationary UV camera, it was possible to illuminate all of the various 1024 pixels of the FPA. Using this procedure, it was found that approximately $95 \%$ of the pixels were active (UV light sensitive), although some variation in pixel responsivity was noted from the raw-data display.

It should be emphasized that the visible-blind UV camera that has been demonstrated is NOT a solar-blind camera. In fact, it is a very sensitive detector of the sun's radiation in the $320-365 \mathrm{~nm}$ UV radiation band. To illustrate this, the camera was taken outside and pointed at the sun. It was found that the sun was easily imaged onto the nitride FPA -- but ND 4 neutral density filters had to be employed to prevent pixel saturation! This again attests to the sensitivity of this new visibleblind UV camera.

\section{Summary and Conclusions.}

A visible-blind UV camera based on a $32 \times 32$ array of GaN/AlGaN p-i-n photodiodes has been successfully demonstrated at room temperature. Each of the 1024 photodiodes in the array consists of a base n-type layer of AlGaN:Si ( 20\%), an undoped GaN layer, and a p- type GaN:Mg layer deposited sequentially by MOVPE. Discrete $200 \mu \mathrm{m}$ x $200 \mu \mathrm{m}$ photodiodes, processed from the same wafer as the photodiode array, display spectral responsivities of $0.2 \mathrm{~A} / \mathrm{W}$ at $358 \mathrm{~nm}$, corresponding to an internal quantum efficiency of $82 \%$. A detector figure of merit $\mathrm{R}_{0} \mathrm{~A}=1.5 \times 10^{9} \Omega-\mathrm{cm}^{2}$ was obtained from which the detectivity $\mathrm{D}^{*}=6.1 \times 10^{13} \mathrm{~cm} \mathrm{~Hz}^{1 / 2} \mathrm{~W}^{-1}$ was estimated. This is one of the highest $\mathrm{D}^{*}$ values ever obtained for any semiconductor photodetector operating at any temperature and at any wavelength. The $32 \times 32$ diode array that formed the sensing element in the visible-blind UV camera displayed similar high sensitivity with $\sim 95 \%$ pixel activation.

This UV camera demonstration represents another historical advancement in optoelectronic devices based on III-V nitride materials. It is, indeed, remarkable to us that the outstanding characteristics of the GaN/AlGaN heterostructure $p-i-n$ photodiodes and the diode FPA that we are reporting were obtained using samples that contain $10^{10}$ or more dislocations per $\mathrm{cm}^{2}$ !! The reason why only nitride devices function in the presence of such large dislocation densities remains a matter of speculation [16]. However, dislocation reduction techniques such as epitaxial lateral overgrowth (ELO) or pendioepitaxy [17] [18] [19] [20], if successfully applied to AlGaN layers, hold promise for even more impressive nitride photodiode characteristics in the future.

When fully developed into large-format photodiode arrays, this new type of UV digital camera may be used in many wide-ranging applications including biological agent detection, missile and shellfire detection, atmospheric ozone-level detection, welding imagery, and flame sensing. In addition, large-format UV staring FPAs based on nitride photodiodes may play an important role in obtaining UV images of the stars and other astronomical objects of importance in understanding the creation and evolution of the universe.

\section{ACKNOWLEDGMENTS}

The work at NCSU is being supported by Army Research Office grants DAAH04-95-1-0627 and DAAD19-99-0113 administered by Dr. M. Dutta and by a Defense Advanced Research Projects Agency grant DAAD19-99-0010 under the Solar Blind Detector Program directed by Dr. R. Leheney. Honeywell would like to acknowledge the support and encouragement of Dr. Paul Schreiber at the Air Force Research Laboratories, Wright-Patterson Air ForceBase, Ohio. 


\section{REFERENCES}

[1] F. A. Ponce, MRS Bull. 22, 51-57 (1997).

[2] Shuji Nakamura, Takashi Mukai, Masayuki Senoh , Appl. Phys. Lett. 64, 1687-1689 (1994).

[3] Shuji Nakamura, Masayuki Senoh, Naruhito Iwasa, Shin-ichi Nagahama, Appl. Phys. Lett. 67, 1868-1870 (1995).

[4] S Nakamura, M Senoh, S Nagahama, N Iwasa, T Yamada, T Matsushita, Y Sugimoto, H Kiyoku, Appl. Phys. Lett. 70, 1417-1419 (1997).

[5] Shuji Nakamura, Gerhard Fasol, The Blue Laser Diode - GaN based Light Emitters and Lasers, (Springer-Verlag, Heidelberg, 1997), .

[6] J Edmond, HS Kong, M Leonard, G Bulman, G Negley, Inst. Phys. Conf. Ser. 142, 991 (1996).

[7] I. Akasaki, S. Sota, H. Sakai, T. Tanaka, M. Koike, H. Amano, Electron. Lett. 32, 1105-1106 (1996).

[8] G.E. Bulman, K. Doverspike, S.T. Sheppard, T.W. Weeks, H.S. Kong, H.M. Dieringer, J.A. Edmond, J.D. Brown, J.T. Swindell, J.F. Schetzina, Electron. Lett. 33, 15561557 (1997).

[9] M.P. Mack, A. Abare, M. Aizcorbe, Peter Kozodoy, S. Keller, U. K. Mishra, L. Coldren, Steven DenBaars, MRS Internet J. Nitride Semicond. Res. 2, 41 (1997).

[10] S Nakamura, M Senoh, S Nagahama, N Iwasa, T Yamada, T Matsushita, H Kiyoku, Y Sugimoto, T Kozaki, H Umemoto, M Sano, K Chocho, Appl. Phys. Lett. 72, 211213 (1998).

[11] Dennis K. Wickenden, Zhenchun Huang, D. Brent Mott, and Peter K. Shu, "Development of Gallium Nitride Photoconductive Detectors", Johns Hopkins APL Technical Digest, 18(2) 217 (1997). "The authors describe the properties of GaN photoconductive UV detectors and discuss possible NASA applications of these devices."

[12] Wei Yang, Thomas Novova, Subash Krishnankutty, Robert Torreano, Scott McPherson, Holly Marsh, Appl. Phys. Lett. 73, 1086 (1998).

[13] Hadis Morkoc, Nav. Res. Rev. 51, 26 (1999).

[14] S.L. Chuang, Physics of Optoelectronic Devices, Wiley, New York (1995). "This book contains a very good summary of the theory and properties of photodetectors."

[15] The Book of Photon Tools, p. 1-25, distributed by Oriel Corporation, available through theWorld Wide Web at http:// www.oriel.com. "This is a well-known source catalog for optical components."

[16] M.A.L. Johnson, Zhonghai Yu, J.D. Brown, F.A. Koeck, N.A. El-Masry, H.S. Kong, J.A. Edmond, J.W. Cook, Jr., J.F. Schetzina, MRS Internet J. Nitride Semicond. Res. 4S1, G5.10 (1999).

[17] O-H Nam, MD Bremser, TS Zheleva, RF Davis, Appl. Phys. Lett. 71, 2638-2340 (1997).

[18] Tsvetanka S. Zheleva, Scott A. Smith, Darren B. Thomson, Thomas Gehrke, Kevin J. Linthicum, Pradeep Rajagopal, Eric Carlson, Waeil M. Ashmawi, Robert F. Davis, MRS Internet J. Nitride Semicond. Res. 4S1, G3.38 (1999).

[19] Zhonghai Yu, M.A.L. Johnson, J.D. Brown, N.A. ElMasry, J.F. Muth, J.W. Cook, Jr., J.F. Schetzina, K.W. Haberern, H.S. Kong, J.A. Edmond, MRS Internet J. Nitride Semicond. Res. 4S1, G4.3 (1999).

[20] K. Linthicum, D. Hanser, T. Zheleva, O. K. Nam, R. F. Davis, Nav. Res. Rev. 51, 26 (1999). 


\section{FIGURES}

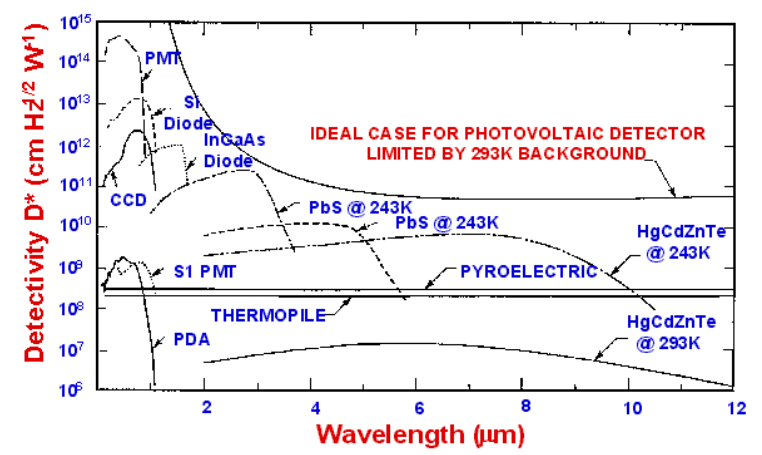

Figure 1. Spectral detectivities for selected photodetectors.
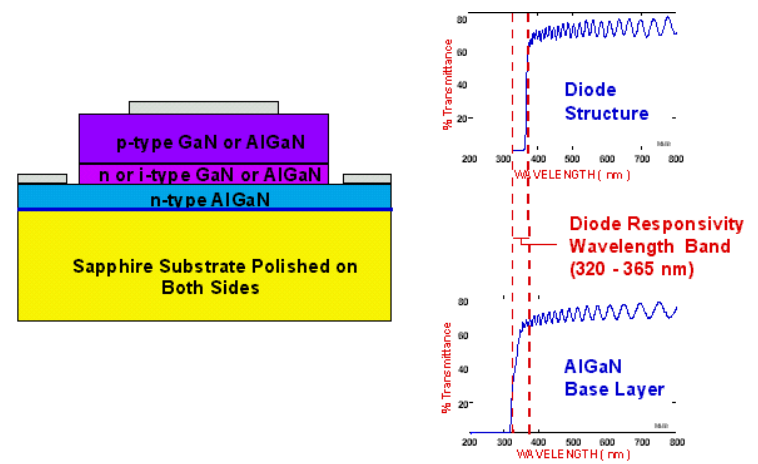

Figure 2. GaN/AlGaN photodiode structure. At the right, optical absorption spectra obtained for the AlGaN and GaN layers of the device are shown to illustrate the UV responsivity band.

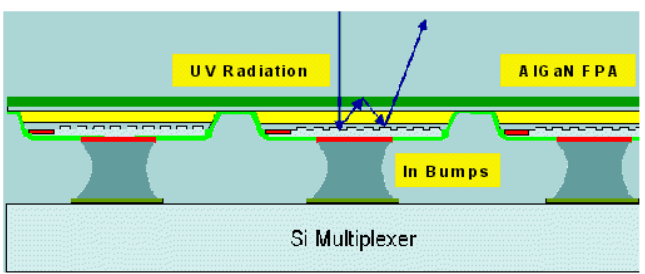

Figure 3. Hybridization process for UV digital camera. 

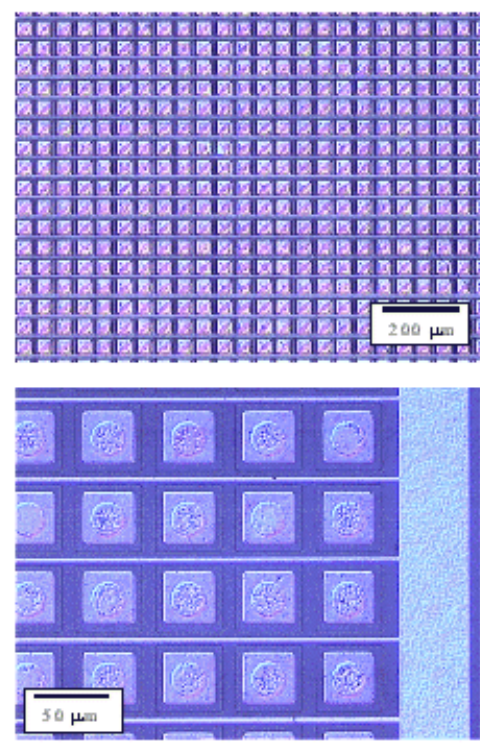

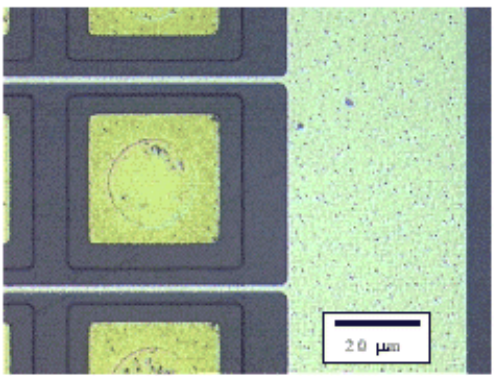

$53.4 \mathrm{\mu m}$ center-to-c enter $m$ es a distance

$44 \mu \mathrm{m} \times 44 \mu \mathrm{m}$ etched mesas

$35 \mu \mathrm{m} \times 35 \mu \mathrm{m}$-metal bond pa ds

$24 \mu \mathrm{m}$ diameter in dium bump pads

20 fully processed arrays at II C SU

Figure 4. Nomarski photographs of a 32 x $32 \mathrm{GaN} / \mathrm{AlGaN}$ photodiode array.
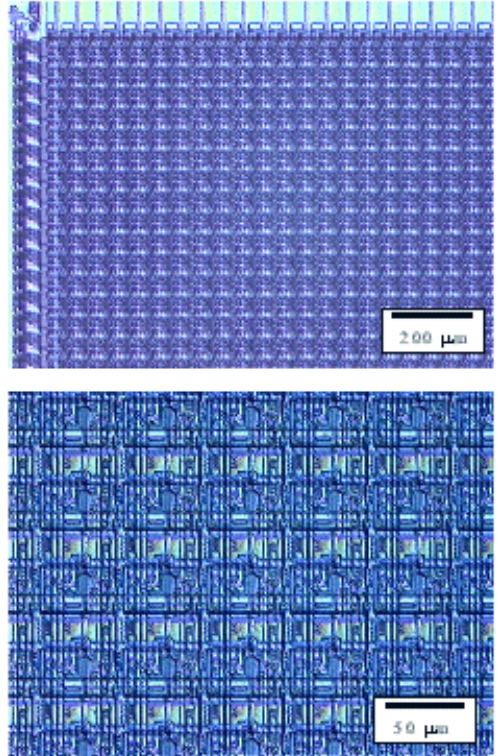

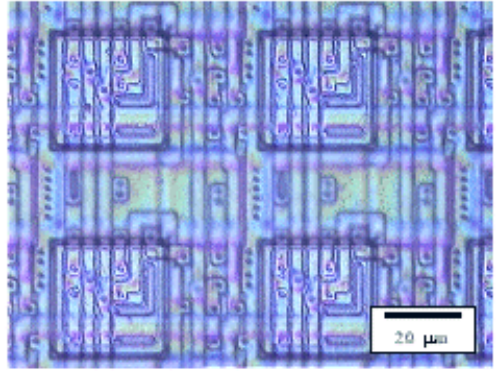

$53.4 \mathrm{~mm}$ center-to-center pixel distance

$24 \mu \mathrm{m} \times 24 \mu \mathrm{m} m$ etal bond $p$ ads

24 um diameter in dium bump pads

36 readout devices for $32 \times 32$ diode arrays availab le at NCSU and NVL

- Imaging hardware/so ftware in place

Figure 5. Nomarski photographs of a 32 x 32 silicon ROIC. 


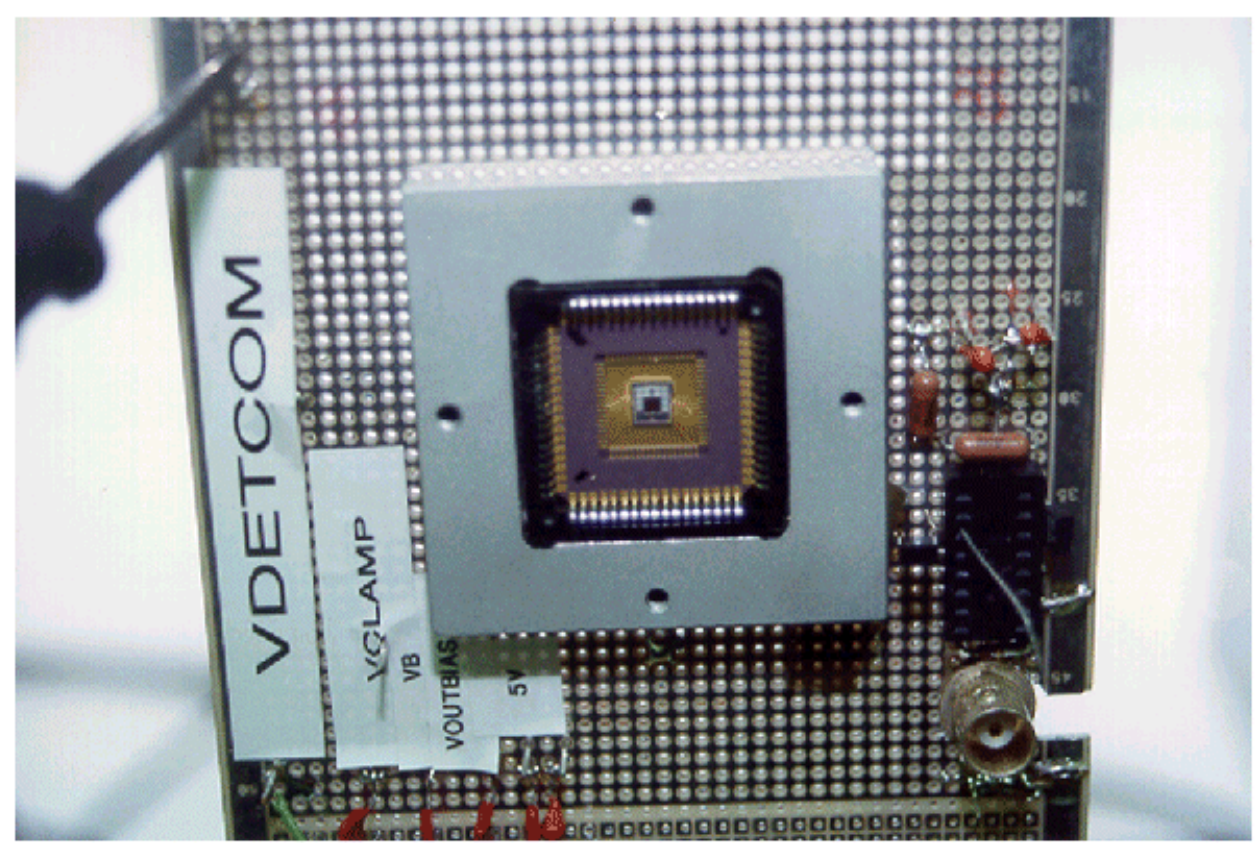

Figure 6. Hybridized GaN/AlGaN UV photodiode array mounted on PC board prior to testing .

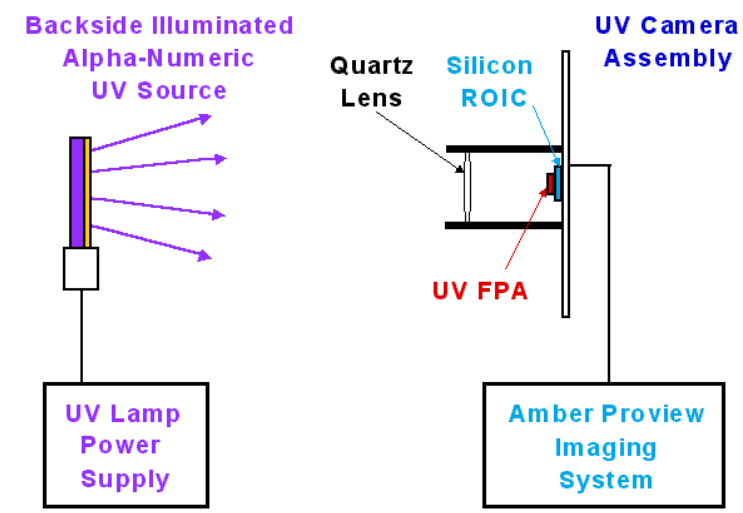

Figure 7. Experimental setup used at NVL to evaluate the visible-blind UV digital camera. 


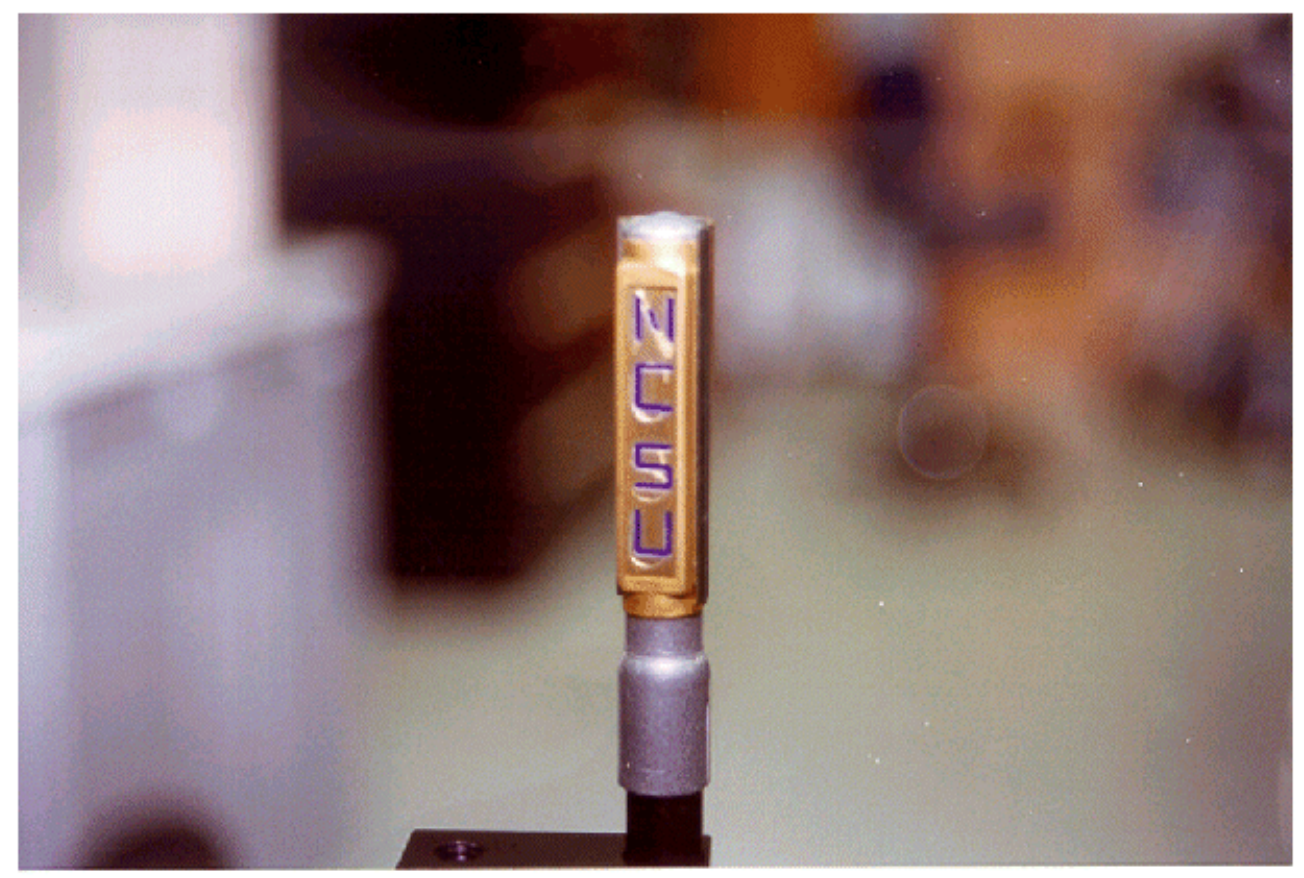

Figure 8. Back-lighted UV alpha-numeric ("NCSU") source used for nitride FPA testing at NVL.

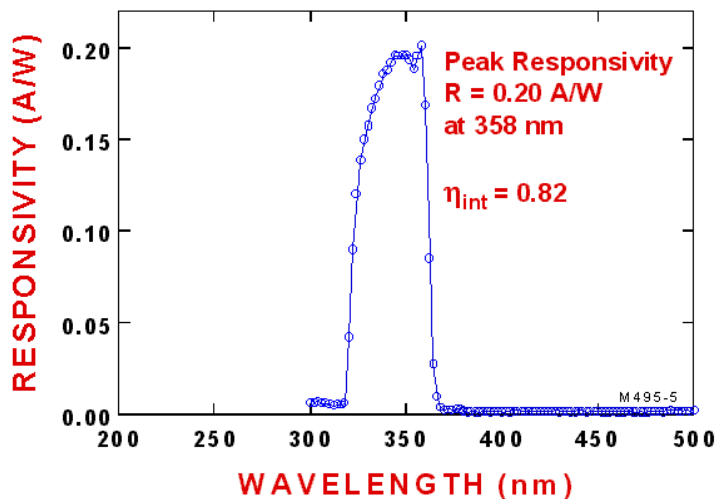

Figure 9. Spectral responsivity of discrete $(200 \mu \mathrm{m} \times 200 \mu \mathrm{m})$ $\mathrm{GaN} / \mathrm{AlGaN}$ heterostructure photodiode operating in the photovoltaic mode (zero bias).
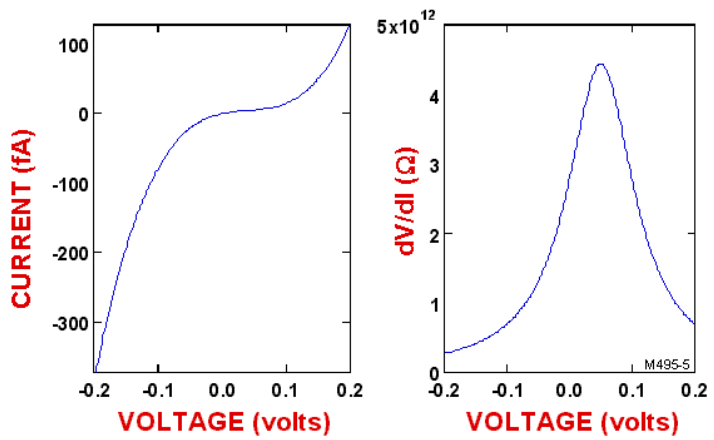

Figure 10. Current-voltage characteristics of a discrete GaN/ AlGaN p-i-n heterostructure photodiode (left). The dynamic resistance versus voltage is also shown (right). Note that the diode dynamic resistance peaks near zero-bias and is very large. This implies high optical detectivity. $\mathrm{R}_{0} \mathrm{~A}=1.5 \times 10^{9} \Omega-$ $\mathrm{cm}^{2}$ and $\mathrm{D}^{*}=6.1 \times 10^{13} \mathrm{~cm} \mathrm{~Hz}^{1 / 2} \mathrm{~W}^{-1}$ were obtained for this device. 


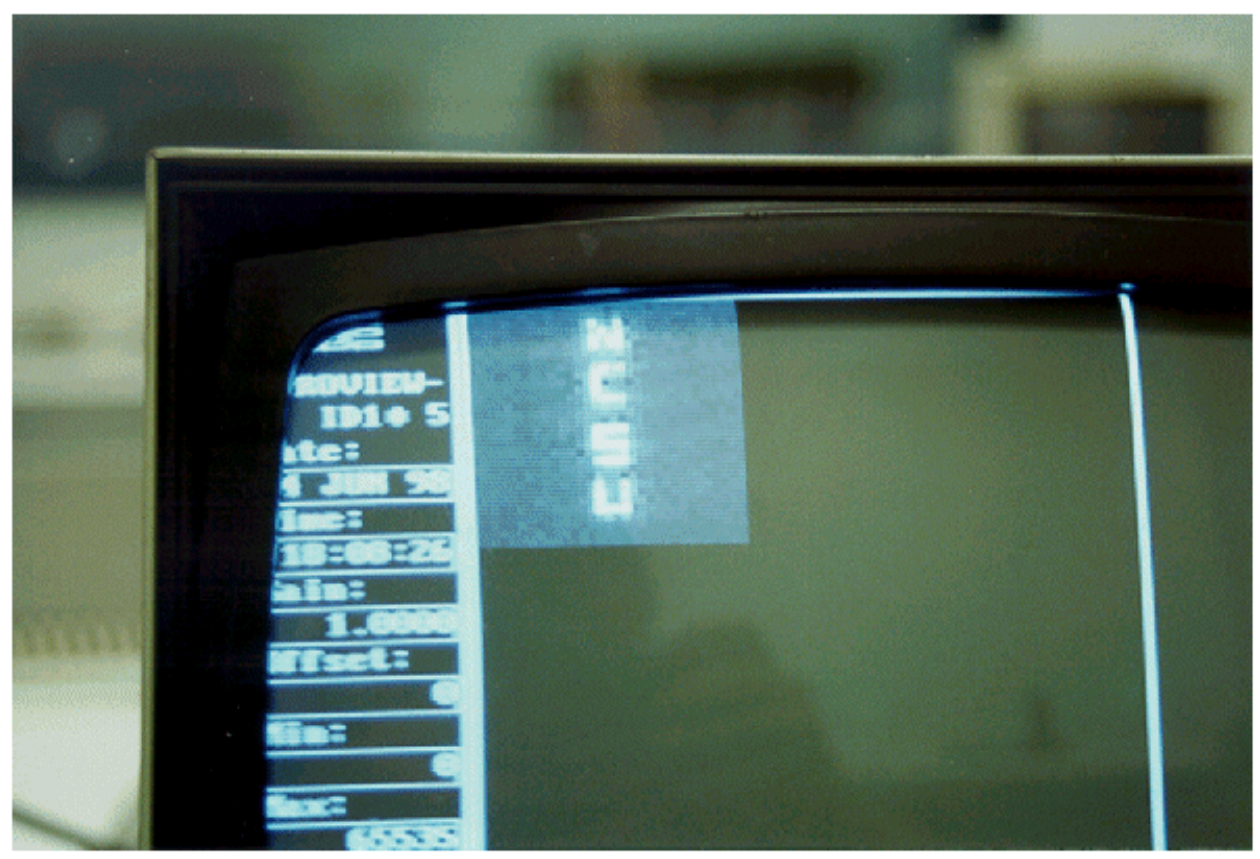

Figure 11. First demonstration of a visible-blind UV image from a III-V nitride-based photodiode array. The image from the FPA was recorded at NVL on July 28, 1999.
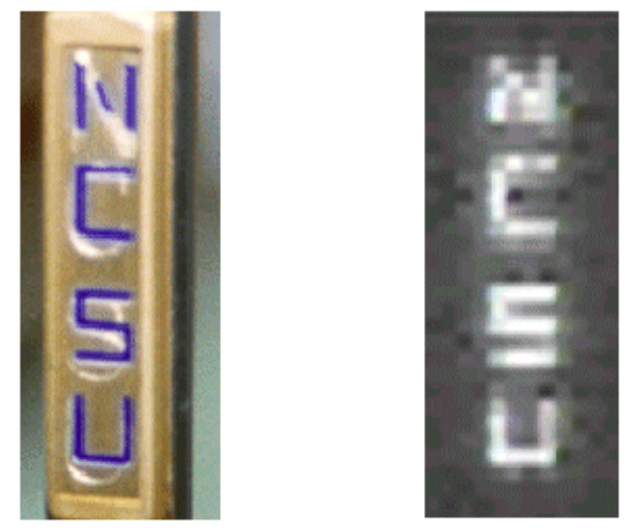

Figure 12. Comparison of UV source (left) with digital image generated by the GaN/AlGaN focal plane array of visible-blind UV sensitive photodiodes (right). 